\title{
Designs of Compact, Flexible, Directive, Near-Field Resonant Parasitic (NFRP) Antennas
}

\author{
Ming-Chun Tang, Xiaoming Chen, Yunlu Duan, Ting Shi, \\ and Mei Li \\ College of Communication Engineering, Chongqing \\ University, Chongqing, 400044, China \\ E-mail: tangmingchun@cqu.edu.cn
}

\begin{abstract}
The designs of compact, low-profile, planar, flexible, directive, quasi-Yagi antennas are presented. By placing near-field resonant parasitic (NFRP) elements around the basic driven dipoles, these NFRP antennas achieve compactness, high efficiency and high directivity. The NFRP elements act either as director or reflector elements, empowering the antenna with desirable quasi-Yagi performance characteristics. These NFRP antennas are fabricated using thin substrates which can be bent without enduring any structure damage. The flexibility of these antennas is investigated under two bending conditions by mounting them on different radii cylinders. These antennas can be used in many advanced applications such as intelligent transportation system (ITS) and wearable devices.
\end{abstract}

Keywords- Compact antennas, directive antennas, flexible antennas, near-field resonant parasitic elements, quasi-Yagi antennas

\section{INTRODUCTION}

Compact directive antennas play an important role in modern wireless communication systems. Their advantages include reducing multi-path fading effects, attractive high directivities, and miniaturized sizes. Many methods have been reported to realize compact-sized high-directivity antennas. One uses large metal ground or electromagnetic band gap (EBG) structures [1]. A second introduces multiple near-field resonant parasitic (NFRP) elements for directivity enhancement [2]. A third combines electric dipoles together with magnetic dipoles to realize Huygens source antennas [34]. A fourth arranges the antenna elements to arrive at a quasiYagi antenna or an end-fire array configuration [5-6]. These systems also have the advantage of being simple and low cost designs. Moreover, when their adaptability to a variety of sophisticated physical environment is considered, antennas with flexibilities highlight their unique merits [7]. For instance, antennas equipped on the high-speed mobile platforms and vehicles are required to be conformal to the surface on which they reside to ensure they meet critical criteria, e.g., aerodynamic performance and other mechanical requirements [8].

In this paper, two types of compact, flexible, quasi-Yagi antennas are reported and analyzed. Their geometries and performance characteristics are illustrated in Section II and Section III. Their flexibility is studied and discussed in Section IV. Finally, some conclusions are drawn in Section V.

\author{
Richard W. Ziolkowski \\ University of Technology Sydney, Global Big Data \\ Technologies Centre, Ultimo NSW 2007, Australia \\ E-mail: Richard. Ziolkowski@uts.edu.au
}

\section{CPW-FED PRINTED FLEXIBLE UNIPLANER QUASI-YAGI ANTENNAS}

The layout of the CPW-fed planar flexible quasi-Yagi antenna is shown in Fig. 1. It is fabricated on a $0.127 \mathrm{~mm}$ thick, Rogers RT/Duroid 5880 substrate whose relative dielectric constant $\varepsilon_{r}=2.2$ and loss tangent $\tan \delta=0.0009$. The antenna comprises a driven semi-loop strip, an arc-shaped strip, and ground strips. This semi-loop strip is CPW-fed with one end connected to the inner conductor of the SMA and the other end connected to the ground strip. The driven strip and ground strips are connected to the inner and outer conductor of the SMA, respectively. The arc-shaped strip, serving as the NFRP element, is placed separate from the semi-loop strip. The measured and simulated performance characteristics of this antenna are depicted in Figs. 2 and 3. The antenna is resonant at $1.56 \mathrm{GHz}$ and its $-10-\mathrm{dB}$ impedance bandwidth is $79.0 \mathrm{MHz}$. Its maximum realized gain was $5.76 \mathrm{dBi}$, and the front-to-back ratio (FTBR) is $12.67 \mathrm{~dB}$. It has quite good directional radiation performance. Because its length is slightly shorter than the overall length of the two ground strips, it will act as the director element while the ground strip acts as a reflector element, respectively. Consequently, the radiation pattern of quasi-Yagi antenna oriented along the $+z$-axis.

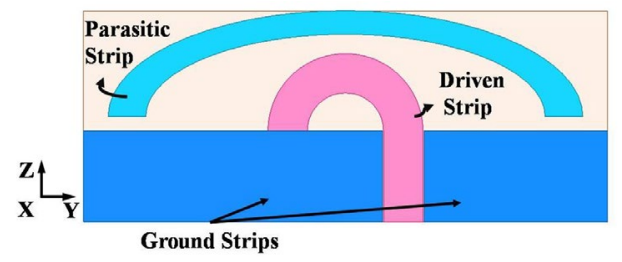

Fig. 1. The geometry of the CPW-fed printed flexible quasi-Yagi uniplanar antenna [9].

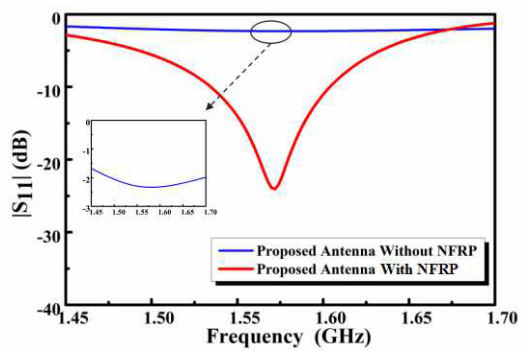

Fig. 2. The reflection coefficient results of the flexible quasi-Yagi antenna and the corresponding antenna without the NFRP element. [9] 


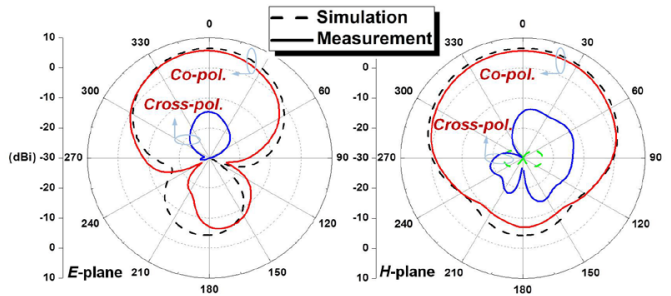

Fig. 3. Measured (simulated) 2-D co-pol and cross-pol gain radiation patterns at $1.56 \mathrm{GHz}(1.58 \mathrm{GHz})$ of the CPW-fed printed flexible quasi-Yagi uniplanar antenna [9].

\section{A. Backward radiation performance}

In order to understand more completely its operating mechanisms, this uni-planar quasi-Yagi antenna was retuned to radiate in the opposite endfire direction (-z-axis). The arcstrip length was set longer than that of the ground strips. As illustrated in Fig. 4, the NFRP element and the ground strips are now acting as the reflector and director elements.

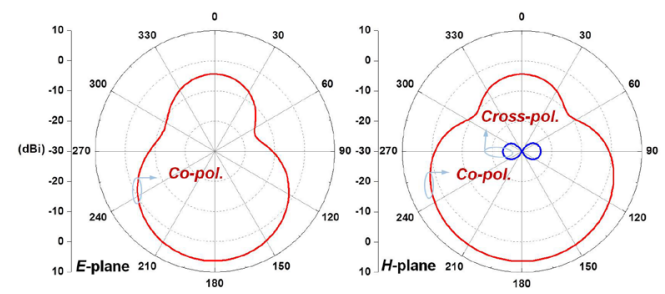

Fig. 4. Co-pol and cross-pol gain radiation patterns at $1.58 \mathrm{GHz}$ when the CPW-fed printed flexible quasi-Yagi uniplanar antenna is in backwarddirected configuration.[9]

\section{B. Further Miniaturizaiton}

To further reduce the antenna's electrical size, several evolutions/modifications were carried out. Five same-sized rectangular slots were cut from the ground strips in order to achieve meander-line ground strips. The NFRP arc strip was also bent to further decrease the size of its footprint. This combination reduced the overall size of the antenna. The final geometry is shown in Fig. 5(a). Its total size is $k a=0.973$. The simulated reflection coefficients of this antenna are shown in Fig. 5(b). The antenna is resonant at $1.574 \mathrm{GHz}$ and a $-10-\mathrm{dB}$ impedance bandwidth of $32.4 \mathrm{MHz}(\sim 2.1 \%)$ is obtained. The maximum gain was $5.26 \mathrm{dBi}$, and the FTBR was $14.58 \mathrm{~dB}$.

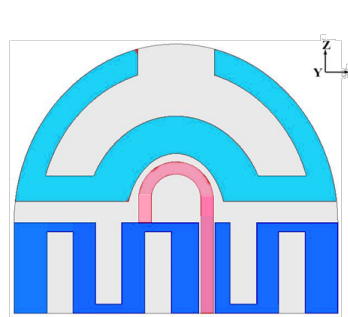

(a)

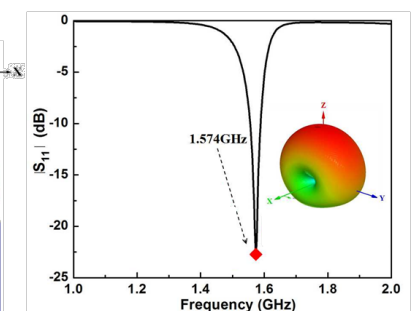

(b)
Fig. 5. Electrically small flexible antenna. (a) Geometry. (b) Reflection coefficient $\left(\left|\mathrm{S}_{11}\right|\right.$ values) as a function of the source frequency together with the 3-D directivity radiation pattern at the resonance frequency. [10]

\section{COMPACT RECONFIGURABLE FLEXIBLE NFRP ANTENNA}

The pattern-reconfigurable flexible NFRP antenna was elaborately designed according to reconfigurable technology
[11], as shown in Fig. 6(a). The antenna comprises a pair of coax-fed driven dipole elements and a pair of NFRP elements. As shown in Figs. 6(b) and (c), four PIN diodes are integrated with the driven dipoles. These dipoles are controlled by the PIN diodes configuration (State R or State L); and as a result, the antenna is pattern-reconfigurable. When the antenna is operating in these two states, two end-fire radiation patterns that point in diametrically opposite directions are obtained. These two NFRP elements act as the director and reflector elements, respectively, for either pattern-reconfigurable state.

As shown in Fig. 7, the antenna is resonant at two frequency points: $1.708 \mathrm{GHz}$ and $1.798 \mathrm{GHz}$, and a fractional $-10-\mathrm{dB}$ impedance bandwidth of $13.4 \%$ is obtained. The simulated impedance bandwidth results are in good agreement with the measured ones. The measured maximum realized gain, radiation efficiency, and FTBR values are $4.43 \mathrm{dBi}, 70.3 \%$, and $13.56 \mathrm{~dB}$, respectively. As illustrated in Fig. 8, the radiation pattern of the antenna in State $\mathrm{R}$ (right) is end-fire and oriented along the $+y$-axis. Vice versa, the radiation pattern oriented to the $-y$-axis when antenna operates in State L (left).

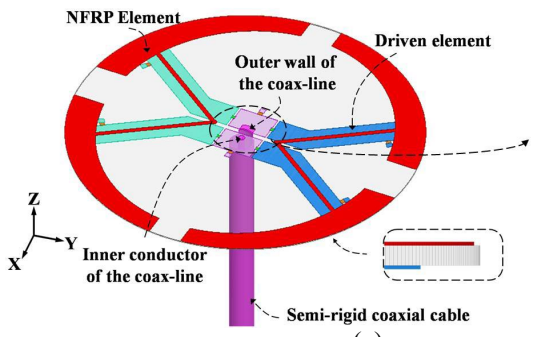

(a)

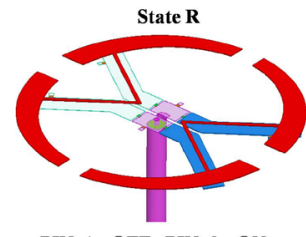

PIN_1 : OFF PIN_3 : ON PIN_2 : OFF PIN_4 : ON

(b)

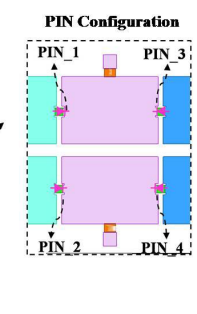

State L

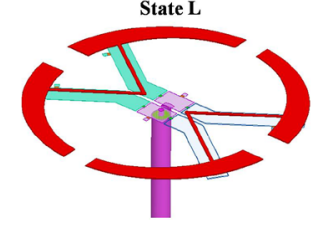

PIN_1 : ON PIN_3 : OFF PIN_2 : ON PIN_4 : OFF

(c)
Fig. 6. Pattern-reconfigurable, flexible, NFRP antenna. (a) the 3-D view and arrangement of four PIN-diodes. (b) State R configuration. (c) State L configuration. [12]

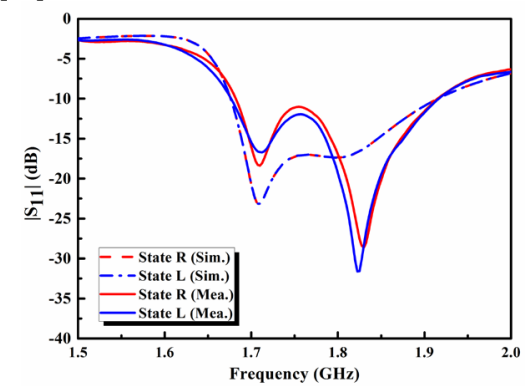

Fig. 7. The measured and simulated reflection coefficient results $\left(\left|S_{11}\right|\right.$ values $)$ of the flexible pattern-reconfigurable antenna in State R and State L. [12]

\section{FLEXIBILITY STUDY}

The flexibility of these antennas is achieved due to the thin substrate and is investigated by mounting them on Styrofoam or other low dielectric constant cylinders with different radii. The compact pattern-reconfigurable NFRP design was 
measured when mounting on Styrofoam cylinder, as shown in Fig. 9. The measured impedance bandwidth, gain, FTBR, and radiation efficiency of its $x$ - and $y$-axis bending configurations are compared in Table I. It is readily concluded that bending in both the $x$ - and $y$-axis directions has little influence on the antenna's performance.

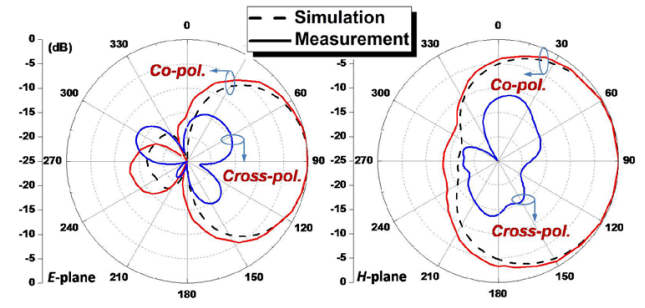

Fig. 8. The realized gain pattern of the pattern-reconfigurable flexible NFRP antenna at its lower resonance frequency in its State $\mathrm{R}$.

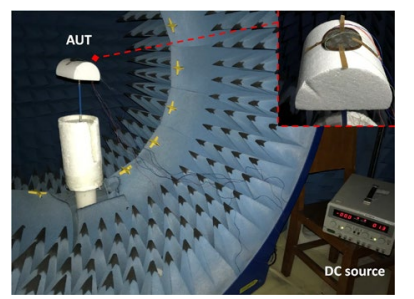

Fig. 9. The prototype antenna in section III as the AUT in the chamber

TABle I. MEASUREd Performances of COMPACt RECONFIGURABle FLEXIBLE NFRP ANTENNA WHEN IT IS MOUNTED CONFORMALLY ON CYlinders Under Two BENDING CONDITIONS WiTH DifFERENT RADII

(a) Bending with respect to the $y$-axis (parallel to the cylinder axis)

\begin{tabular}{|c|c|c|c|c|}
\hline \multirow{2}{*}{ Bending Condition } & $50 \mathrm{~mm}$ & $70 \mathrm{~mm}$ & $90 \mathrm{~mm}$ & $110 \mathrm{~mm}$ \\
\cline { 2 - 5 } & 14.5 & 14.6 & 13.4 & 13.2 \\
\hline $\begin{array}{c}\text { Fractional } \\
\text { Bandwidth (\%) }\end{array}$ & 3.9 & 3.98 & 4.00 & 3.92 \\
\hline $\begin{array}{c}\text { Maximum } \\
\text { Realized Gain } \\
(\mathrm{dB})\end{array}$ & 67.6 & 66.8 & 67.9 & 67.3 \\
\hline $\begin{array}{c}\text { Radiation } \\
\text { Efficiency (\%) }\end{array}$ & 12.71 & 12.9 & 13.52 & 13.4 \\
\hline $\begin{array}{c}\text { Maximum FTBR } \\
(\mathrm{dB})\end{array}$ & & & & \\
\hline
\end{tabular}

(b) Bending with respect to the $x$-axis (perpendicular to the cylinder axis)

\begin{tabular}{|c|c|c|c|c|}
\hline \multirow{2}{*}{ Bending Condition } & $50 \mathrm{~mm}$ & $70 \mathrm{~mm}$ & $90 \mathrm{~mm}$ & $110 \mathrm{~mm}$ \\
\cline { 2 - 5 } & 14.3 & 13.4 & 13.1 & 13.2 \\
\hline $\begin{array}{c}\text { Fractional } \\
\text { Bandwidth (\%) }\end{array}$ & 3.79 & 3.86 & 4.22 & 3.82 \\
\hline $\begin{array}{c}\text { Maximum Realized } \\
\text { Gain (dB) }\end{array}$ & 62.8 & 65.4 & 63.00 & 63.54 \\
\hline $\begin{array}{c}\text { Radiation } \\
\text { Efficiency (\%) }\end{array}$ & 9.72 & 9.97 & 11.31 & 13.62 \\
\hline $\begin{array}{c}\text { Maximum FTBR } \\
(\mathrm{dB})\end{array}$ & & & \\
\hline
\end{tabular}

\section{V.CONCLUSION}

Two types of compact, flexible, directional NFRP antennas were presented. It was demonstrated that the placement of a NFRP element near to a dipole antenna improves the antenna's directivity. In these designs, the NFRP elements have either a director or reflector role in the resulting quasiYagi configurations. These reported antennas exhibit good end-fire radiation performance together with high FTBR values whether they radiate in their planar or either bending conditions.

\section{Acknowledgments}

This work was supported in part by the National Natural Science Foundation of China contract number 61471072 and 61701052 , in part by the Funding of the Innovative Leading Talents in Science and Technology of Chongqing contract number CSTCCXLJRC201705, in part by the Funding of the leading research talent cultivation plan of Chongqing University contract number cqu2017hbrc1A08, and in part by Funding of the Young Backbone Teachers in Colleges and Universities of Chongqing contract number 0307001104102, in part by the Fundamental Research Funds for the Central Universities contract number 2018CDQYTX0025, and in part by the Australian Research Council grant number DP160102219.

\section{References}

[1] J. Ng, and R. W. Ziolkowski, "Combining metamaterial-inspired electrically small antennas with electromagnetic band gap (EBG) structures to achieve higher directivities and bandwidths," in Proc. 2012 International Workshop on Antenna Technology (iWAT 2012), Tucson, America, pp. 189-192, Mar. 2012.

[2] R. W. Ziolkowski, M.-C. Tang, and N. Zhu, "An efficient, broad bandwidth, high directivity, electrically small antenna," Microw. Opt. Technol. Lett., vol. 55, no. 6, pp. 1430-1434, Jun. 2013.

[3] M.-C. Tang, H. Wang, and R. W. Ziolkowski, "Design and testing of simple, electrically small, low-profile, Huygens source antennas with broadside radiation performance," IEEE Trans. Antennas Propag., vol.64, no. 11, pp. 4607-4617, Nov. 2016.

[4] M. -C. Tang, T. Shi, and R. W. Ziolkowski, "Electrically small, broadside radiating Huygens source antenna augmented with internal non-Foster elements to increase its bandwidth," IEEE Antennas Wireless Propag. Lett., vol.16, pp. 712-715, 2017.

[5] M.-C. Tang, R.W. Ziolkowski, S. Xiao, and M. Li, "A highdirectivity, wideband, efficient, electrically small antenna system," IEEE Trans.Antennas Propag., vol. 62, no. 12, pp. 6541-6547, Dec. 2014.

[6] A. D. Yaghjian, T. H. O’Donnell, E. E. Altshuler, and S. R. Best, "Electrically small supergain end-fire arrays," Radio Sci., vol. 43, no. 3, pp. 1-13, 2008.

[7] S. Hong, S. H. Kang, Y. Kim, and C. W. Jung, "Transparent and flexible antenna for wearable glasses applications," IEEE Trans. Antennas Propag., vol. 64, no. 7, pp. 2797-2804, Jul. $2016 .$.

[8] B. D. Braaten, S. Roy, S. Nariyal, M. A. Aziz, N. F. Chamberlain, I. Irfanullah, M. T. Reich, and D. E. Anagnostou, "A self-adapting flexible (SELFLEX) antenna array for changing conformal surface applications," IEEE Trans. Antennas Propag., pp. 655-665, vol. 61 no. 2, Feb. 2013.

[9] M.-C. Tang, T. Shi, and R. W. Ziolkowski, "Flexible efficient quasiYagi printed uniplanar antenna," IEEE Trans. Antennas Propag., vol. 63, no. 12, pp. 5343-5350, Dec. 2015.

[10] M.-C. Tang, B. Y. Zhou, and R. W. Ziolkowski, "Flexible uniplanar electrically small directive antenna empowered by a modified CPWfeed," IEEE Antennas Wireless Propag. Lett., vol. 15, pp. 914-917, 2016.

[11] M.-C. Tang, H. Wang, T. Deng, and R. W. Ziolkowski, "Compact planar ultra-wideband antennas with continuously tunable, independent band-notched filters," IEEE Trans. Antennas Propag., vol.64, no. 8, pp. 3292-3301, Aug. 2016.

[12] M.-C. Tang, B. Y. Zhou, Y. Duan, X. Chen and R. W. Ziolkowski, "Pattern-reconfigurable, flexible, wideband, directive, electrically small near-field resonant parasitic antenna" IEEE Trans. Antennas Propag., vol. 66, no. 5, pp. 2271-2280, May 2018. 